\title{
A Quantitative Estimate for the Sampling Kantorovich Series in Terms of the Modulus of Continuity in Orlicz Spaces
}

\author{
DANILO COSTARELLI* AND GIANLUCA VINTI
}

\begin{abstract}
In the present paper we establish a quantitative estimate for the sampling Kantorovich operators with respect to the modulus of continuity in Orlicz spaces defined in terms of the modular functional. At the end of the paper, concrete examples are discussed, both for what concerns the kernels of the above operators, as well as for some concrete instances of Orlicz spaces.
\end{abstract}

Keywords: Sampling Kantorovich series, Orlicz spaces, Modulus of continuity, Quantitative estimates, Kernels.

2010 Mathematics Subject Classification: 41A25, 41A05, 41A35, 46E30, 47A58, $47 \mathrm{~B} 38$.

\section{INTRODUCTION}

The sampling Kantorovich operators $S_{w}$ have been introduced by Bardaro, Butzer, Stens and Vinti in [8], in order to study an $L^{1}$-version of the so-called generalized sampling operators $([12,32,14])$. The main peculiarity of the sampling Kantorovich operators is that they revealed to be suitable in order to reconstruct not necessarily continuous signals ([2]).

Indeed, in the original paper [8] the authors proved the modular convergence of the operators $S_{w}$ in the general setting of Orlicz spaces, which include, as a special case, the $L^{p}$-spaces.

Later on, the operators $S_{w}$ have been studied under different aspects, both from theoretical $([17,5,23])$ and applications point of view $([6,7])$. For instance, in $[6,7]$ some applications to energy engineering have been developed applying an algorithm for image reconstruction and enhancement based on the multivariate version of the operators $S_{w}$ for the processing of thermographic images.

The order of approximation for the sampling Kantorovich operators has been also studied in [21]; this has been done assuming the function $f$ in suitable Lipschitz classes, both in the space of uniformly continuous and bounded functions (i.e., in $C(\mathbb{R})$ ) and in Orlicz spaces (i.e., in $L^{\varphi}(\mathbb{R})$ ). For other results concerning the order of approximation for the above operators, see, e.g., [31, 11].

The above problem has been faced in $C(\mathbb{R})$ also from the quantitative point of view in [9], by using the modulus of continuity of the function being approximated.

Currently, the study of quantitative estimates in the setting of Orlicz spaces in terms of the modulus of continuity is still an open problem.

For the latter reason, in this paper we establish the quantitative rate of convergence for the sampling Kantorovich operators; in order to do this we firstly recall the notion of the modulus of continuity in $L^{\varphi}(\mathbb{R})$ which is based on the modular functional of the space ([10]).

Received: 17 November 2018; Accepted: 4 December 2018; Published Online: 4 January 2019

*Corresponding author: D. Costarelli; danilo.costarelli@unipg.it

DOI: $10.33205 / \mathrm{cma} .484500$ 
At the end of the paper, several examples of kernels and concrete cases of Orlicz spaces are recalled. For instance, the $L^{p}$-spaces, with $1 \leq p<+\infty$, are included in the present general theory, together with other well-known examples of Orlicz spaces.

\section{NOTATION AND PRELIMINARIES}

We begin this section by recalling some basic facts concerning Orlicz spaces.

A function $\varphi: \mathbb{R}_{0}^{+} \rightarrow \mathbb{R}_{0}^{+}$is said to be a $\varphi$-function if it satisfies the following conditions: $(\Phi 1) \varphi$ is a non decreasing and continuous function;

$(\Phi 2) \varphi(0)=0, \varphi(u)>0$ if $u>0$ and $\lim _{u \rightarrow+\infty} \varphi(u)=+\infty$.

Let us now consider the functional $I^{\varphi}$ associated to the $\varphi$-function $\varphi$ and defined by

$$
I^{\varphi}[f]:=\int_{\mathbb{R}} \varphi(|f(x)|) d x,
$$

for every $f \in M(\mathbb{R})$, i.e., for every (Lebesgue) measurable function $f: \mathbb{R} \rightarrow \mathbb{R}$. As it is wellknown, $I^{\varphi}$ is a modular functional (see e.g. [29, 10]), and the Orlicz space generated by $\varphi$ is defined by

$$
L^{\varphi}(\mathbb{R}):=\left\{f \in M(\mathbb{R}): I^{\varphi}[\lambda f]<\infty, \text { for some } \lambda>0\right\} .
$$

A notion of convergence in Orlicz spaces, called modular convergence, was introduced in [30].

We will say that a net of functions $\left(f_{w}\right)_{w>0} \subset L^{\varphi}(\mathbb{R})$ is modularly convergent to $f \in L^{\varphi}(\mathbb{R})$, if there exists $\lambda>0$ such that

$$
I^{\varphi}\left[\lambda\left(f_{w}-f\right)\right]=\int_{\mathbb{R}} \varphi\left(\lambda\left|f_{w}(x)-f(x)\right|\right) d x \longrightarrow 0, \quad w \rightarrow+\infty .
$$

Moreover we recall, for the sake of completeness, that in $L^{\varphi}(\mathbb{R})$ it can be also given a strong notion of convergence, i.e. the Luxemburg-norm convergence, see e.g. $[29,10]$. We will say that a net of functions $\left(f_{w}\right)_{w>0} \subset L^{\varphi}(\mathbb{R})$ is convergent to $f \in L^{\varphi}(\mathbb{R})$ with respect to the Luxemburg norm if (2.1) holds for every $\lambda>0$. Definition (2.1) induces a topology in $L^{\varphi}(\mathbb{R})$, called modular topology. Obviously, the modular convergence and the Luxemburg norm convergence coincide if and only if the well-known $\Delta_{2}$-condition on $\varphi$ is satisfied, see, e.g., $[29,10]$.

Now, we recall the definition of the modulus of continuity in Orlicz spaces $L^{\varphi}(\mathbb{R})$, with respect to the modular $I^{\varphi}$. For any fixed $f \in L^{\varphi}(\mathbb{R})$, and for a suitable $\lambda>0$, we denote:

$$
\omega(f, \delta)_{\varphi}:=\sup _{|t| \leq \delta} I^{\varphi}[\lambda(f(\cdot+t)-f(\cdot))],
$$

with $\delta>0$.

For general references concerning Orlicz spaces and some of their generalizations, see, e.g., $[28,1,24,25,18]$.

In order to define the considered operators, we need some additional notions.

Let $\Pi=\left(t_{k}\right)_{k \in \mathbb{Z}}$ be a sequence of real numbers such that $-\infty<t_{k}<t_{k+1}<+\infty$ for every $k \in \mathbb{Z}, \lim _{k \rightarrow \pm \infty} t_{k}= \pm \infty$ and there are two positive constants $\Delta, \delta$ such that $\delta \leq \Delta_{k}:=$ $t_{k+1}-t_{k} \leq \Delta$, for every $k \in \mathbb{Z}$.

In what follows, a function $\chi: \mathbb{R} \rightarrow \mathbb{R}$ will be called a kernel if it satisfies the following properties:

- $(\chi 1) \chi \in L^{1}(\mathbb{R})$ and is bounded in a neighborhood of 0 ;

- $(\chi 2)$ for every $u \in \mathbb{R}$

$$
\sum_{k \in \mathbb{Z}} \chi\left(u-t_{k}\right)=1
$$


- $(\chi 3)$ for some $\beta>0$,

$$
m_{\beta, \Pi}(\chi):=\sup _{u \in \mathbb{R}} \sum_{k \in \mathbb{Z}}\left|\chi\left(u-t_{k}\right)\right| \cdot\left|u-t_{k}\right|^{\beta}<+\infty .
$$

Then, the sampling Kantorovich operators $S_{w}$ for a given kernel $\chi$ are defined by:

$$
\left(S_{w} f\right)(x):=\sum_{k \in \mathbb{Z}} \chi\left(w x-t_{k}\right)\left[\frac{w}{\Delta_{k}} \int_{t_{k} / w}^{t_{k+1} / w} f(u) d u\right] \quad(x \in \mathbb{R}),
$$

where $f: \mathbb{R} \rightarrow \mathbb{R}$ is a locally integrable function such that the series is convergent for every $x \in \mathbb{R}$.

There holds the following lemma.

Lemma 2.1 ([8]). Under the assumptions $(\chi 1)$ and $(\chi 3)$ on the kernel $\chi$, it turns out:

$$
m_{0, \Pi}(\chi):=\sup _{u \in \mathbb{R}} \sum_{k \in \mathbb{Z}}\left|\chi\left(u-t_{k}\right)\right|<+\infty .
$$

Note that, it is easy to see that the discrete absolute moment $m_{0, \Pi}(\chi)>0$.

\section{THE MAIN RESULT}

We can prove the following quantitative estimate for the sampling Kantorovich operators by using the modulus of continuity in Orlicz spaces.

Theorem 3.1. Let $\varphi$ be a convex $\varphi$-function. Suppose that, for any fixed $0<\alpha<1$, we have:

$$
w \int_{|y|>1 / w^{\alpha}}|\chi(w y)| d y \leq M w^{-\gamma}, \quad \text { as } w \rightarrow+\infty,
$$

for suitable positive constants $M, \gamma$ depending on $\alpha$ and $\chi$. Then, for $f \in L^{\varphi}(\mathbb{R})$, and $\lambda>0$ there holds:

$$
\begin{aligned}
I^{\varphi}\left[\lambda\left(S_{w} f-f\right)\right] & \leq \frac{\|\chi\|_{1}}{2 \delta m_{0, \Pi}(\chi)} \omega\left(2 m_{0, \Pi}(\chi) f, \frac{1}{w^{\alpha}}\right)_{\varphi} \\
& +\frac{M I^{\varphi}\left[4 \lambda m_{0, \Pi}(\chi) f\right]}{2 \delta m_{0, \Pi}(\chi)} w^{-\gamma}+\frac{\Delta}{2 \delta} \omega\left(2 m_{0, \Pi}(\chi) f, \frac{1}{w}\right)_{\varphi},
\end{aligned}
$$

for every sufficiently large $w>0$, where $m_{0, \Pi}(\chi)<+\infty$ in view of Lemma 2.1. In particular, if $\lambda>0$ is sufficiently small, the above inequality implies the modular convergence of the sampling Kantorovich operators $S_{w} f$ to $f$.

Proof. Let $\lambda>0$ be fixed. Using the convexity of $\varphi$, and since $\varphi$ is non decreasing, we can write what follows:

$$
\begin{gathered}
I^{\varphi}\left[\lambda\left(S_{w} f-f\right)\right] \\
\leq \frac{1}{2}\left\{\int_{\mathbb{R}} \varphi\left(2 \lambda\left|\left(S_{w} f\right)(x)-\sum_{k \in \mathbb{Z}} \chi\left(w x-t_{k}\right) \frac{w}{\Delta_{k}} \int_{t_{k} / w}^{t_{k+1} / w} f\left(u+x-t_{k} / w\right) d u\right|\right) d x\right. \\
\left.+\int_{\mathbb{R}} \varphi\left(2 \lambda\left|\sum_{k \in \mathbb{Z}} \chi\left(w x-t_{k}\right) \frac{w}{\Delta_{k}} \int_{t_{k} / w}^{t_{k+1} / w} f\left(u+x-t_{k} / w\right) d u-f(x)\right|\right) d x\right\}=: I_{1}+I_{2},
\end{gathered}
$$


$w>0$. We estimate $I_{1}$. By using the Jensen inequality (see, e.g., [19]) twice, and the change of variable $y=x-t_{k} / w$, we obtain:

$$
\begin{aligned}
& 2 I_{1} \leq \int_{\mathbb{R}} \varphi\left(2 \lambda \sum_{k \in \mathbb{Z}}\left|\chi\left(w x-t_{k}\right)\right| \frac{w}{\Delta_{k}} \int_{t_{k} / w}^{t_{k+1} / w}\left|f(u)-f\left(u+x-t_{k} / w\right)\right| d u\right) d x \\
& \leq \frac{1}{m_{0, \Pi}(\chi)} \int_{\mathbb{R}} \sum_{k \in \mathbb{Z}}\left|\chi\left(w x-t_{k}\right)\right| \varphi\left(2 \lambda m_{0, \Pi}(\chi) \frac{w}{\Delta_{k}} \int_{t_{k} / w}^{t_{k+1} / w}\left|f(u)-f\left(u+x-t_{k} / w\right)\right| d u\right) d x \\
& \leq \frac{1}{m_{0, \Pi}(\chi)} \int_{\mathbb{R}} \sum_{k \in \mathbb{Z}}\left|\chi\left(w x-t_{k}\right)\right| \frac{w}{\Delta_{k}} \int_{t_{k} / w}^{t_{k+1} / w} \varphi\left(2 \lambda m_{0, \Pi}(\chi)\left|f(u)-f\left(u+x-t_{k} / w\right)\right|\right) d u d x \\
& \leq \frac{\delta^{-1}}{m_{0, \Pi}(\chi)} \int_{\mathbb{R}}|\chi(w y)| w \sum_{k \in \mathbb{Z}} \int_{t_{k} / w}^{t_{k+1} / w} \varphi\left(2 \lambda m_{0, \Pi}(\chi)|f(u)-f(u+y)|\right) d u d y \\
& =\frac{\delta^{-1}}{m_{0, \Pi}(\chi)} \int_{\mathbb{R}}|\chi(w y)| w \int_{\mathbb{R}} \varphi\left(2 \lambda m_{0, \Pi}(\chi)|f(u)-f(u+y)|\right) d u d y \\
& =\frac{\delta^{-1}}{m_{0, \Pi}(\chi)} \int_{\mathbb{R}} w|\chi(w y)| I^{\varphi}\left[2 \lambda m_{0, \Pi}(\chi)(f(\cdot)-f(\cdot+y))\right] d y=: J,
\end{aligned}
$$

$w>0$. Let now $0<\alpha<1$ be fixed. Thus we can split the above integral $J$ as follows:

$$
\begin{gathered}
J:=\frac{w \delta^{-1}}{m_{0, \Pi}(\chi)} \times \\
\left\{\int_{|y| \leq 1 / w^{\alpha}}+\int_{|y|>1 / w^{\alpha}}\right\}|\chi(w y)| I^{\varphi}\left[2 \lambda m_{0, \Pi}(\chi)(f(\cdot)-f(\cdot+y))\right] d y=: J_{1}+J_{2} .
\end{gathered}
$$

For $J_{1}$, we have:

$$
\begin{aligned}
J_{1} & \leq \frac{w \delta^{-1}}{m_{0, \Pi}(\chi)} \int_{|y| \leq 1 / w^{\alpha}}|\chi(w y)| \omega\left(2 m_{0, \Pi}(\chi) f,|y|\right)_{\varphi} d y \\
& \leq \omega\left(2 m_{0, \Pi}(\chi) f, 1 / w^{\alpha}\right)_{\varphi} \frac{w \delta^{-1}}{m_{0, \Pi}(\chi)} \int_{|y| \leq 1 / w^{\alpha}}|\chi(w y)| d y \\
& \leq \omega\left(2 m_{0, \Pi}(\chi) f, 1 / w^{\alpha}\right)_{\varphi} \frac{\delta^{-1}\|\chi\|_{1}}{m_{0, \Pi}(\chi)},
\end{aligned}
$$

$w>0$. Moreover, by using the convexity of $\varphi$, for $J_{2}$ we can obtain:

$$
\begin{aligned}
J_{2} \leq \frac{w \delta^{-1}}{m_{0, \Pi}(\chi)} \int_{|y|>1 / w^{\alpha}}|\chi(w y)| \frac{1}{2}\left\{I^{\varphi}\left[4 \lambda m_{0, \Pi}(\chi) f\right]\right. \\
\left.+I^{\varphi}\left[4 \lambda m_{0, \Pi}(\chi) f(\cdot+y)\right]\right\} d y .
\end{aligned}
$$

Obviously, it is easy to see that:

$$
I^{\varphi}\left[4 \lambda m_{0, \Pi}(\chi) f\right]=I^{\varphi}\left[4 \lambda m_{0, \Pi}(\chi) f(\cdot+y)\right],
$$

for every $y$. Then, by exploiting assumption (3.4), we finally obtain:

$$
\begin{aligned}
J_{2} & \leq \frac{w \delta^{-1}}{m_{0, \Pi}(\chi)} \int_{|y|>1 / w^{\alpha}}|\chi(w y)| I^{\varphi}\left[4 \lambda m_{0, \Pi}(\chi) f\right] d y \\
& \leq \frac{\delta^{-1}}{m_{0, \Pi}(\chi)} I^{\varphi}\left[4 \lambda m_{0, \Pi}(\chi) f\right] M w^{-\gamma}
\end{aligned}
$$

for $w>0$ sufficiently large. 
Now, we can estimate $I_{2}$. Using Jensen inequality twice (as above), the change of variable $y=u-t_{k} / w$, and Fubini-Tonelli theorem, we have:

$$
\begin{aligned}
& \leq \frac{1}{m_{0, \Pi}(\chi)} \int_{\mathbb{R}} \sum_{k \in \mathbb{Z}}\left|\chi\left(w x-t_{k}\right)\right| \frac{w}{\Delta_{k}} \int_{t_{k} / w}^{t_{k+1} / w} \varphi\left(2 \lambda m_{0, \Pi}(\chi)\left|f\left(u+x-t_{k} / w\right)-f(x)\right|\right) d u d x \\
& \leq \frac{\delta^{-1}}{m_{0, \Pi}(\chi)} \int_{\mathbb{R}} \sum_{k \in \mathbb{Z}}\left|\chi\left(w x-t_{k}\right)\right| w \int_{0}^{\Delta / w} \varphi\left(2 \lambda m_{0, \Pi}(\chi)|f(x+y)-f(x)|\right) d y d x \\
& \leq \delta^{-1} \int_{\mathbb{R}} w \int_{0}^{\Delta / w} \varphi\left(2 \lambda m_{0, \Pi}(\chi)|f(x+y)-f(x)|\right) d y d x \\
& \leq \delta^{-1} w \int_{0}^{\Delta / w} I^{\varphi}\left[2 \lambda m_{0, \Pi}(\chi)(f(\cdot+y)-f(\cdot))\right] d y \\
& \leq \delta^{-1} \omega\left(2 m_{0, \Pi}(\chi) f, 1 / w\right)_{\varphi} w \int_{0}^{\Delta / w} d y=\delta^{-1} \Delta \omega\left(2 m_{0, \Pi}(\chi) f, 1 / w\right)_{\varphi},
\end{aligned}
$$

$w>0$. This completes the proof.

Remark 3.1. Note that, it is easy to show that for any kernels such that $\chi(u)=\mathcal{O}\left(|u|^{-\theta}\right)$, as $|u| \rightarrow+\infty$, for $\theta>1$, we have that assumption (3.4) is satisfied for some constant $M>0$ and $\gamma=(1-\alpha)(\theta-1)>0$, for every fixed $0<\alpha<1$.

\section{EXAMPLES}

Examples of convex $\varphi$-functions generating remarkable Orlicz spaces, where the above result is valid are:

$\varphi_{p}(u):=u^{p}, 1 \leq p<\infty, \varphi_{\alpha, \beta}:=u^{\alpha} \log ^{\beta}(u+e)$, for $\alpha \geq 1, \beta>0$ and $\varphi_{\gamma}(u)=e^{u^{\gamma}}-1$, for $\gamma>0, u \geq 0$. It is well-known that $\varphi_{p}$ generates the $L^{p}(\mathbb{R})$-space and the corresponding convex modular functional is given by $I^{\varphi_{p}}[f]:=\|f\|_{p}^{p}$, while $\varphi_{\alpha, \beta}$ and $\varphi_{\gamma}$ generate the $L^{\alpha} \log ^{\beta} L$-spaces (or Zygmund spaces), largely used, e.g., in the theory of partial differential equations, and the exponential spaces respectively, e.g., used for embedding theorems between Sobolev spaces. The convex modular functionals corresponding to $\varphi_{\alpha, \beta}$ and $\varphi_{\gamma}$ are

$$
I^{\varphi_{\alpha, \beta}}[f]:=\int_{\mathbb{R}}|f(x)|^{\alpha} \log ^{\beta}(e+|f(x)|) d x, \quad(f \in M(\mathbb{R})),
$$

and

$$
I^{\varphi_{\gamma}}[f]:=\int_{\mathbb{R}}\left(e^{|f(x)|^{\gamma}}-1\right) d x, \quad(f \in M(\mathbb{R}))
$$

respectively.

Now, we give a brief list of some well-known and important class of kernels which satisfy the above assumptions $(\chi 1)-(\chi 3)$, and for which Theorem 3.1 holds.

First of all, we recall the definition of the well-known central B-spline of order $N$ (see e.g., $[33,3,4])$ :

$$
\beta^{N}(x):=\frac{1}{(N-1) !} \sum_{i=0}^{N}(-1)^{i}\left(\begin{array}{c}
N \\
i
\end{array}\right)\left(\frac{N}{2}+x-i\right)_{+}^{N-1}, \quad x \in \mathbb{R} .
$$

It is well-known that $\beta^{N}$ have compact support, then (3.4) is obviously satisfied for every $\gamma>0$. 
Other important (band-limited) kernels are given by the so-called Jackson type kernels of order $N$, defined by:

$$
J_{N}(x):=c_{N} \operatorname{sinc}^{2 N}\left(\frac{x}{2 N \pi \alpha}\right), \quad x \in \mathbb{R},
$$

with $N \in \mathbb{N}, \alpha \geq 1$, and $c_{N}$ is a non-zero normalization coefficient, given by:

$$
c_{N}:=\left[\int_{\mathbb{R}} \operatorname{sinc}^{2 N}\left(\frac{u}{2 N \pi \alpha}\right) d u\right]^{-1} .
$$

For $J_{N}$, assumption (3.4) turns out to be satisfied in view of what has been observed in Remark 3.1. For the sake of completeness, we recall that the well-known (above mentioned) sinc-function is that defined as $\sin (\pi x) / \pi x$, if $x \neq 0$, and 1 if $x=0$, see e.g., [26, 27]. For other examples of kernels, see, e.g., [13, 20, 15, 22, 16].

\section{ACKNOWLEDGMENTS}

The authors are members of the Gruppo Nazionale per l'Analisi Matematica, la Probabilitá e le loro Applicazioni (GNAMPA) of the Istituto Nazionale di Alta Matematica (INdAM).

The authors are partially supported by the "Department of Mathematics and Computer Science" of the University of Perugia (Italy). Moreover, the first author has been partially supported within the 2018 GNAMPA-INdAM Project entitled: "Dinamiche non autonome, analisi reale e applicazioni", while the second author within the project "Metodi di Teoria dell'Approssimazione, Analisi Reale, Analisi Nonlineare e loro applicazioni", funded by the 2018 basic research fund of the University of Perugia.

\section{REFERENCES}

[1] A. Abdurexit and T. N. Bekjan: Noncommutative Orlicz modular spaces associated with growth functions. Banach J. Math. Anal. 9 (4) (2015), 115-125.

[2] T. Acar, A. Alotaibi and S. A. Mohiuddine: Construction of new family of Bernstein-Kantorovich operators. Math. Methods Appl. Sci. 40 (18) (2017), 7749-7759.

[3] G. Allasia, R. Cavoretto and A. De Rossi: A class of spline functions for landmark-based image registration, Math. Methods Appl. Sci. 35 (8) (2012), 923-934.

[4] G. Allasia, R. Cavoretto and A. De Rossi: Lobachevsky spline functions and interpolation to scattered data, Comput. Appl. Math. 32 (1) (2013), 71-87.

[5] L. Angeloni, D. Costarelli and G. Vinti: A characterization of the convergence in variation for the generalized sampling series. Ann. Acad. Sci. Fenn. Math. 43 (2018), 755-767.

[6] F. Asdrubali, G. Baldinelli, F. Bianchi, D. Costarelli, A. Rotili, M. Seracini and G. Vinti: Detection of thermal bridges from thermographic images by means of image processing approximation algorithms, Appl. Math. Comp. 317 (2018), $160-171$.

[7] F. Asdrubali, G. Baldinelli, F. Bianchi, D. Costarelli, L. Evangelisti, A. Rotili, M. Seracini and G. Vinti: A model for the improvement of thermal bridges quantitative assessment by infrared thermography. Applied Energy 211 (2018), 854-864.

[8] C. Bardaro, P. L. Butzer, R. L. Stens and G. Vinti: Kantorovich-type generalized sampling series in the setting of Orlicz spaces. Sampl. Theory Signal Image Process. 6 (1) (2007), 29-52.

[9] C. Bardaro and I. Mantellini: On convergence properties for a class of Kantorovich discrete operators. Num. Funct. Anal. Optim. 33 (4) (2012), 374-396.

[10] C. Bardaro, J. Musielak and G. Vinti: Nonlinear Integral Operators and Applications. De Gruyter Series in Nonlinear Analysis and Applications, 9 New York, Berlin, 2003.

[11] B. Bartoccini, D. Costarelli and G. Vinti: Extension of saturation theorems for the sampling Kantorovich operators. In print in: Complex Analysis and Operator Theory (2018), DOI: 10.1007/s11785-018-0852-z.

[12] P. L. Butzer: A survey of the Whittaker-Shannon sampling theorem and some of its extensions, J. Math. Res. Exposition 3 (1) (1983), 185-212.

[13] P. L. Butzer and R. J. Nessel: Fourier Analysis and Approximation, Vol. I: One-dimensional theory, Pure and Applied Mathematics, 40, Academic Press, New York-London, 1971. 
[14] P. L. Butzer, S. Ries and R. L. Stens: Approximation of Continuous and Discontinuous Functions by Generalized Sampling Series. J. Approx. Theory 50 (1) (1987), 25-39.

[15] L. Coroianu and S. G. Gal: $L^{p}$-approximation by truncated max-product sampling operators of Kantorovich-type based on Fejér kernel. J. Integral Equations Appl. 29 (2) (2017), 349-364.

[16] L. Coroianu and S. G. Gal: Approximation by truncated max-product operators of Kantorovich-type based on generalized ( $\Phi, \Psi)$-kernels. Math. Methods Appl. Sci. 41 (2018), 7971-7984.

[17] D. Costarelli, A.M. Minotti and G. Vinti: Approximation of discontinuous signals by sampling Kantorovich series. J. Math. Anal. Appl. 450 (2) (2017), 1083-1103.

[18] D. Costarelli and A.R. Sambucini: Approximation results in Orlicz spaces for sequences of Kantorovich max-product neural network operators. Results Math. 73 (1) (2018), Art. 15, 15 pp. DOI: 10.1007/s00025-018-0799-4.

[19] D. Costarelli and R. Spigler: How sharp is the Jensen inequality ?, J. Inequal. Appl. 2015:69 (2015) 1-10.

[20] D. Costarelli and G. Vinti: Approximation by Nonlinear Multivariate Sampling-Kantorovich Type Operators and Applications to Image Processing. Numer. Funct. Anal. Optim. 34 (8) (2013), 819-844.

[21] D. Costarelli and G. Vinti: Order of approximation for sampling Kantorovich operators, J. Integral Equations Appl. 26 (3) (2014), 345-368.

[22] D. Costarelli and G. Vinti: Convergence for a family of neural network operators in Orlicz spaces. Math. Nachr. 290 (2-3) (2017), 226-235.

[23] D. Costarelli and G. Vinti: An inverse result of approximation by sampling Kantorovich series. Proceedings of the Edinburgh Mathematical Society, 62 (1) (2019), 265-280.

[24] D. Cruz-Uribe and P. Hasto: Extrapolation and interpolation in generalized Orlicz spaces. Trans. Amer. Math. Soc. 370 (6) (2018), 4323-4349.

[25] P. A. Hasto: The maximal operator on generalized Orlicz spaces. J. Funct. Anal. 269 (12) (2015), $4038-4048$.

[26] Y. S. Kolomoitsev and M. A. Skopina: Approximation by multivariate Kantorovich-Kotelnikov operators. J. Math. Anal. Appl. 456 (1) (2017), 195-213.

[27] A. Krivoshein and M. A. Skopina: Multivariate sampling-type approximation, Anal. Appl. 15 (4) (2017), $521-542$.

[28] K. Kuaket and P. Kumam: Fixed points of asymptotic pointwise contractions in modular spaces. Appl. Math. Lett. 24 (11) (2011), 1795-1798.

[29] J. Musielak: Orlicz spaces and Modular Spaces. Lecture Notes in Math. 1034 Springer-Verlag, Berlin, 1983.

[30] J. Musielak and W. Orlicz: On modular spaces. Studia Math. 18 (1959), 49-65.

[31] O. Orlova and G. Tamberg: On approximation properties of generalized Kantorovich-type sampling operators. J. Approx. Theory 201 (2016), 73-86.

[32] S. Ries and R. L. Stens: Approximation by generalized sampling series. In: Proc. Internat. Conf. Constructive Theory of Functions, Varna, Bulgaria, June 1984, pp. 746-756, Bulgarian Acad. Sci. Sofia, 1984.

[33] M. Unser: Ten good reasons for using spline wavelets. Proc. SPIE Vol. 3169, Wavelets Applications in Signal and Image Processing V (1997), 422-431.

UNIVERSITY OF PERUGIA

Department of MATHEMATICS AND COMPUter SCIENCE

1, Via VANVITElli, 06123 PERUGia, ITAly

E-mail address: danilo. costarelli@unipg.it

UNIVERSITY OF PERUGIA

DEPARTMENT OF MATHEMATICS AND COMPUTER SCIENCE

1, Via VANVitelli, 06123 Perugia, Italy

E-mail address: gianluca.vinti@unipg.it 